cardiometabolic side effects including weight gain with a risk of Type 2 Diabetes Mellitus. There is limited understanding of the factors increasing susceptibility to these side effects. It is established that increased adiposity associated with weight gain is mediated by the emergence of a persistent low-grade inflammatory state. However, there has been no research investigating the relationships between pro-inflammatory states in children and the cardiometabolic side-effects of SGAs and there are no clinical indicators of those at risk.

Aims

1. To determine whether there is a subgroup of patients at baseline who present with a profile of immune dysregulation.

2. To investigate how SGA medication impacts on inflammatory markers and cardiometabolic function in children.

3. To investigate the potential to predict those at greater risk of developing adverse metabolic outcomes in response to the treatment with SGAs.

Methods We are recruiting children and adolescents (5-18 years) who are commencing SGA medication. We assess the cardiometabolic profile clinically and biochemically and obtain serum and peripheral blood mononuclear cells (PBMCs) to measure levels of inflammatory markers. Through comparison with biobanked healthy control samples, we will determine if there is a subgroup at baseline with a pro-inflammatory profile. The patient groups are assessed longitudinally at 3,6 and 12 months to measure BMI percentile and cardiometabolic function. Changes in immune cells and inflammatory markers are measured including IL-1, IL-10, IL-17, sCD-163, TNF- $\alpha$ and IFN- $\gamma$ as well as leptin, ghrelin and adiponectin in response to treatment with antipsychotics.

Results Twelve patients have been recruited and are being followed up longitudinally. Of the data analysis that has been completed, it appears that leptin and TNF- $\alpha$ levels have increased in the participants with the most weight gain $(6-9 \mathrm{~kg})$ between baseline and 3 months after commencing SGAs.

Discussion Identification of a high-risk group for weight gain is vitally important and it would allow clinicians to work with these patients to minimise the metabolic side effects of the medications; through rational SGA choice, promotion of intensive monitoring and implementation of preventative treatment regimes. Antipsychotic use is a prominent cause of obesity in young people with mental health disorders. Through this study, we aim to modify clinical practice, which we hope will lead to better guidelines to reduce the risks.

\section{P627 MITOCHONDRIAL DISEASE MIMICS}

\footnotetext{
'Samantha Doyle, 'Zaza Abidin, 'Suranga Senanayake, 'Stephanie James, ${ }^{2}$ Mei Yap, ${ }^{3}$ Caroline Hart, ${ }^{3,4}$ Ellen Crushell, ${ }^{5}$ Shane Smyth, ${ }^{6,7}$ Andrew Green, ${ }^{1,8}$ Eileen Treacy, ${ }^{2}$ Tim Lynch, 'Gregory Pastores, ${ }^{2}$ Aoife Laffan, 'James O'Byrne*. 'National Centre for Inherited Metabolic Disorders, Mater Misericordiae University Hospital, Dublin, Ireland; ${ }^{2}$ Department of Neurology, Dublin Neurological Institute, Mater Misericordiae University Hospital, Dublin, Ireland; ${ }^{3}$ National Centre for Inherited Metabolic Disorders, Temple Street Children's University Hospital, Dublin, Ireland; ${ }^{4}$ University College Dublin, Belfield, Dublin, Ireland; ${ }^{5}$ Department of Neurology, Mater Misericordiae University Hospital, Dublin, Ireland; ${ }^{6}$ Department of Clinical Genetics, Our Lady's Children's Hospital, Crumlin, Dublin, Ireland; ${ }^{7}$ School of Medicine and Medical Science, University College Dublin, Dublin, Ireland; ${ }^{8}$ Department of Paediatrics, Trinity College, Dublin, Ireland
}

10.1136/archdischild-2019-epa.958
A diagnosis of primary mitochondrial disease was traditionally arrived at on the basis of clinical and biochemical features including abnormal respiratory chain analysis on muscle biopsy and/or identification of other 'mitochondrial disease markers'. With the increased availability of genetic testing, in particular massive parallel sequencing, alternative primary diagnoses which result in secondary mitochondrial dysfunction are being identified

We present a cohort of six cases who previously had a diagnosis of mitochondrial disease. Alternative primary diagnoses have recently been identified which includes AndersenTawil syndrome (gene: KCNJ2), COL4A1-related brain smallvessel disease (gene: COL4A1), cardiofaciocutaneous syndrome (gene: $B R A F$ ), autosomal recessive spinal cerebellar ataxia-10 (gene: ANO10), facioscapulohumeral muscular dystrophy (gene: DUX4) and IGSF1 deficiency syndrome (gene: IGSF1). Conclusion The reported cohort highlights the important point that many genetic conditions may mimic mitochondrial disease and, although the phenotype and biochemical tests may indicate mitochondrial disease, we suggest that genetic confirmation is required to secure a diagnosis. Establishment of an accurate diagnosis is important, not just prognosis and planning of management and treatments regimes, but also for appropriate genetic counseling and the identification of other at-risk family members for possible cascade analysis. The link between many of these primary diagnoses and secondary mitochondrial dysfunction is poorly understood but reporting such cases will allow these pathways to be elucidated and understood.

\section{P628 WILSON'S DISEASE IN CHILDREN: ABOUT 8 CASES}

${ }^{1}$ Manel Hsairi, ${ }^{1}$ Lamia Gargouri, ${ }^{2}$ Chiraz Rgaieg* ${ }^{*}{ }^{1}$ Faiza Safi, ${ }^{1}$ Rania Zribi, ${ }^{2}$ Abdellatif Gargouri, ${ }^{1}$ Abdelmajid Mahfoudh. ${ }^{1}$ Emergency and Pediatric Reanimation Department, Sfax, Tunisia; ${ }^{2}$ Neonatology Department, Sfax, Tunisia

\subsection{6/archdischild-2019-epa.959}

Introduction Wilson's disease is a rare disease characterized by its clinical heterogeneity that causes diagnostic difficulties.

Patients and methods This is a retrospective study in 8 patients with Wilson's disease.

Results The study is about 4 girls and 4 boys. The mean age at diagnosis was 8 years and 8 months. The average diagnostic delay was 58 days. All our patients had liver damage at the time of diagnosis. An anxiodepressive symptomatology was noted in one case. The peri-corneal ring of Kayser-Fleischer was objectified in 2 cases. Hepatocellular insufficiency and cholestasis were observed in 4 cases. Thrombocytopenia was present at the time of diagnosis in 3 cases and hemolytic anemia with Coombs test negative in one case. The genetic study revealed the presence of 2 mutations in the homozygous state in 1 case, 2 mutations in the heterozygous state in 1 case and the absence of mutations in 2 cases. Thanks to the family survey, 2 patients were identified at the presymptomatic stage.

The evolution under treatment with D-penicillamine was favorable for 7 patients. Neurological symptoms occurred in one case. The average decline is 5 years and 4 months.

Conclusion Wilson's disease is a curable genetic condition, its prognosis is all the better when the diagnosis is made in time and as a result the treatment is initiated early. 